\title{
REAL-VALUED FUNCTIONS ON PARTIALLY ORDERED SETS
}

\section{SEYMOUR GINSBURG}

It is known that if $P$ is a partially ordered set, then $P$ can be imbedded into an everywhere branching partially ordered set $Q$ in such a manner that if a function has a limit $L$ on $P$, the function can be extended to $Q$ and have a limit $L$ on $Q .{ }^{1}$ The purpose of this note is to show that $P$ can be imbedded isomorphically into an everywhere branching partially ordered set $Q$ and each function $f$ on $P$ extended to $Q$, in such a manner that $f$ has a limit $L$ on $P$ if and only if it has a $\operatorname{limit} L$ on $Q .^{2}$

By a partially ordered set is meant a set of elements $P=\{p\}$, with a binary relation " $\leqq$ " which has the three properties:

(1) $p \leqq p$ for each element $p$ of $P$;

(2) if $p_{1} \leqq p_{2}$ and $p_{2} \leqq p_{3}$, then $p_{1} \leqq p_{3}$; and

(3) if $p_{1} \leqq p_{2}$ and $p_{2} \leqq p_{1}$, then $p_{1}=p_{2}$ (identity).

As usual, " $p_{1}<p_{2}$ " will mean that $p_{1} \leqq p_{2}$, but $p_{1}$ is not identical with $p_{2}$. An element $p_{0}$ of $P$ is called a minimal (maximal) element of $P$ if there is no element $p$ of $P$ for which $p<p_{0}\left(p>p_{0}\right)$. The only partially ordered sets which are considered hereafter are those which have no minimal elements. A partially ordered set is directed if, for each pair of elements in $P, p_{1}$ and $p_{2}$, an element $p_{3}$ can be found for which $p_{3} \leqq p_{i}, \imath=1,2$. A partially ordered set is everywhere branching if, to each element $p_{1}$ of $P$, there corresponds a pair of elements $p_{2}$ and $p_{3}$ such that $p_{i} \leqq p_{1}, i=2,3$, and

$$
\left\{p \mid p \leqq p_{2}\right\} \cap\left\{p \mid p \leqq p_{3}\right\}=\varnothing
$$

where $\varnothing$ is the empty set. A subset $Q=\{q\}$ of $P$ is a coinitial subset of $P$ if to each element $p$ in $P$, there corresponds an element $q$ in $Q$ such that $q \leqq p . Q=\{q\}$ is a residual subset of $P$ if, for each element $q$ in $Q,\{p \mid p \leqq q, p \in P\}$ is a subset of $Q$.

A single, real-valued function $f$ defined on a partially ordered set $P=\{p\}$ has a limit $L$ if, to each element $p_{0}$ of $P$, and $\epsilon>0$, there corresponds an element $p_{1}\left(p_{0}, \epsilon\right)$ of $P$ such that $p_{1} \leqq p_{0}$ and $|f(p)-L|<\epsilon$ for $p \leqq p_{1} .^{3}$

Presented to the Society, April 26, 1952 under the title Real functions on posets, received by the editors April 7, 1952 and, in revised form, July 15, 1952.

${ }^{1}$ See Day, Oriented systems, Duke Math. J. vol. 11 (1944) p. $201 \mathrm{ff}$.

2 The author wishes to thank the referee for his general suggestions, particularly in the simplification of the proof of Theorem 1.

3 See Alaoglu and, Birkhoff, General ergodic theorems, Ann. of Math. vol. 41 (1940) pp. 293-309. 
A single, real-valued function $f$ defined on a partially ordered set $P$ has a partial limit $L$ on $P$ if, for some residual subset $Q$ of $P$, the function $f$, considered as a function on $Q$, has a limit $L$.

TheоReм 1. A partially ordered set $P=\{p\}$ may be imbedded into an everywhere branching partially ordered set $Q=\{q\}$ by an isomorphism $g^{4}$. Furthermore, a function $h$ of $Q$ onto $g(P)$ can be found which has the following two properties:

(1) $h[g(p)]=g(p)$ for each $p$ in $P$; and

(2) $f$ being any real function on $P$, then the function $f_{*}$, which is defined by (a) $f_{*}[g(p)]=f(p)$ for $p$ in $P$, and (b) $f_{*}(q)=f_{*}[h(q)]$ for $q$ in $Q$, has a limit $L$ on $Q$ if and only if $f$ has a limit $L$ on $P$.

Proof. For each $p$ in $P$ let $g(p)=\{x \mid x \leqq p, x \in P\}$. Let

$$
\{Q=q \mid q \text { is a coinitial subset of } g(p), p \in P\},
$$

and order the elements of $Q$ by set inclusion. To see that $Q$ is an everywhere branching partially ordered set, well order the elements of $P$ into a sequence, say $\left\{r_{\xi}\right\}, \xi<\gamma$. A simply ordered subset $A=\{a\}$ of a partially ordered set $B=\{b\}$ shall be called a path (in $B$ ) if there is no element $b_{0}$ of $B$ such that $b_{0} \leqq a$ for each element $a$ in $A$. Clearly, if $b_{0}$ is any element of $B$, then there exists a path in $B$,

$$
a_{0}>a_{1}>\cdots>a_{\xi}>\cdots \text {. }
$$

Now let $q=\{y\}$ be any element of $Q$. Let $y_{0}^{0}$ be the first element of $q$ and

$$
y_{0}^{0}>y_{1}^{0}>\cdots>y_{\xi}^{0}>\cdots
$$

be any path $Z_{0}$ in $q$ for which $y_{0}^{0}$ is a maximal element. This is possible since $q$ is a partially ordered set with no minimal element. Denote by $A_{0}$ the set

$$
A_{0}=\left\{y \mid y \in q, y \geqq y_{\xi}^{0}, y_{\xi}^{0} \in Z_{0}\right\} .
$$

We continue by transfinite induction. Suppose that the paths $Z_{\mu}$ and the sets $A_{\mu}$ have been defined for $\mu<\lambda$. Let $y_{0}^{\lambda}$ be the first element of $q-\mathrm{U}_{\mu<\lambda} A_{\mu}$. Let

$$
\stackrel{\lambda}{y_{0}}>\stackrel{\lambda}{y_{1}}>\cdots>\hat{y}_{\xi}^{\lambda}>\cdots
$$

be any path $Z_{\lambda}$ in $q$ for which $y_{0}^{\lambda}$ is a maximal element. This is possible since $q-U_{\mu<\lambda} A$ is a partially ordered set with no minimal element.

- A mapping $g$ of a partially ordered set $(P, \leqq 1)$ into a partially ordered set $\left(Q_{1} \leqq^{2}\right)$ is an isomorphism if $g$ is one-to-one, and $p_{1} \leqq^{1} p_{2}$ if and only if $g\left(p_{1}\right) \varliminf^{2} g\left(p_{2}\right)$. 
Denote by $A_{\lambda}$ the set

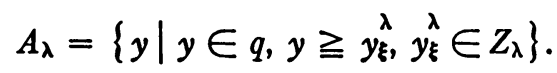

Consider the two subsets of $q$

$$
q_{1}=\left\{y_{\alpha+2 n+1}^{\lambda} \mid \alpha=0 \text { or a limit number, } \lambda \text { any ordinal }\right\}
$$

and

$$
q_{2}=\left\{y_{\alpha+2 n}^{\lambda} \mid \alpha=0 \text { or a limit number, } \lambda \text { any ordinal }\right\} .
$$

Each of the two sets are coinitial in $q$. Therefore $q_{1}$ and $q_{2}$ are elements of $Q$.

Let $g$ be the function which takes each point $p$ of $P$ into the element $g(p)$ of $Q$. Clearly $g$ is an isomorphism of $P$ into $Q$. Let $h$ be the function which is defined by: (1) $h(q)=g\left(p_{0}\right)$ if $p_{0}$ is the unique maximal element of $q$; and (2) $h(q)=g\left(p_{0}\right)$, where $p_{0}$ is the first element of $q$, if $q$ has no unique maximal element. If $f$ is a real function on $P$, then denote by $f_{*}$ the function which is defined by $f_{*}[g(p)]=p$ for $p$ in $P$, and $f_{*}(q)=f_{*}[h(q)]$.

Suppose that $f_{*}$ has a limit $L$ on $Q$. For any element $p_{0}$ of $P$, and $\epsilon>0$, denote by $E_{\epsilon}\left(p_{0}\right)$ the set

$$
E_{\epsilon}\left(p_{0}\right)=\left\{p \mid p \leqq p_{0} \text { and }|f(p)-L| \geqq \epsilon\right\} .
$$

If $E_{\epsilon}\left(p_{0}\right)$ were to be a coinitial subset of $g\left(p_{0}\right)$, then the relation $\left|f_{*}(q)-L\right| \geqq \epsilon$ would be true for all $q \leqq E_{\mathrm{\epsilon}}\left(p_{0}\right)$. But this would contradict the function $f_{*}$ having a limit $L$ on $Q$. Therefore $E_{\epsilon}\left(p_{0}\right)$ is not a coinitial subset of $g\left(p_{0}\right)$. Consequently, for some element $p_{1}$ of $g\left(p_{0}\right)$, i.e., $p_{1} \leqq p_{0}$, we have $\left\{p \mid p \leqq p_{1}\right\} \cap E_{\epsilon}\left(p_{0}\right)=\varnothing$. Hence $|f(p)-L|<\epsilon$ for $p \leqq p_{1}$. Thus $f$ has a limit $L$ on $P$.

Now suppose that the function $f$ has a limit $L$ on $P$. Let $q_{0}$ be any element of $Q$, and $\epsilon>0$. Let $p_{0}$ be any element of $q_{0}$. For some element $p_{1}$ of $P$, where $p_{1} \leqq p_{0}$, we have $|f(p)-L|<\epsilon$ for $p \leqq p_{1}$. If $q_{1}=\left\{p \mid p \leqq p_{1}, p \in q_{0}\right\}$, then $q_{1}$ is a coinitial subset of $g\left(p_{1}\right)$. Thus $q_{1}$ is an element of $Q$. If $q \leqq q_{1}$, then $\left|f_{*}(q)-L\right|<\epsilon$. Therefore the function $f_{*}$ has a limit $L$ on $Q$.

When the partially ordered set $P$ is directed, one can study the behavior on a coinitial subset of $P$ of a real function $f$ defined on $P$, by inspecting the everywhere branching partially ordered set $Q$ and the function $f_{*}$ which are obtained from the previous theorem. Specifically we have

Theorem 2. Let $P$ be a directed partially ordered set and $f$ a real function defined on it. Let $Q$ and $f_{*}$ be the same as in Theorem 1. 
Then a necessary and sufficient condition that $f$ have a limit $L$ on some coinitial subset $q$ of $P$ is that $f_{*}$ have a partial limit $L$ on $Q$.

Proof. The necessity is trivial. Consider the sufficiency. Let $Z$ be a residual subset of $Q$ on which $f_{*}$ has a limit $L$. Let $q_{0}$ be an element of $Z$. $q_{0}$ is a coinitial subset of $P$. Let $\epsilon>0$ and $p_{0}$ be any element of $q_{0}$. Let

$$
q_{1}=\left\{p \mid p \leqq p_{0}, p \in q_{0}\right\} .
$$

Suppose that for each point $p_{1}$ of $q_{1}$, a point $p_{2}$ of $q_{1}$, where $p_{2} \leqq p_{1}$, can be found so that $\left|f\left(p_{2}\right)-L\right| \geqq \epsilon$. Since $P$ is directed, and thus also $q_{0}$ and $q_{1}$,

$$
q_{2}=\left\{p\left|p \in q_{1},\right| f(p)-L \mid \geqq \epsilon\right\}
$$

is a coinitial subset of $P$. Furthermore, $\left|f_{*}(q)-L\right| \geqq_{\epsilon}$ for $q \leqq q_{1}$. Thus $f_{*}$ cannot have the limit $L$ on $Z$. From this contradiction we see that for some $p_{1} \leqq p_{0},|f(p)-L|<\epsilon$ for $p \leqq p_{1}$. Consequently $f$ has the limit $L$ on $q_{0}$.

UNIVERSITY OF MIAMI 